\title{
Long Noncoding RNA HOTAIRM1 Maintains Tumorigenicity of Glioblastoma Stem-Like Cells Through Regulation of HOX Gene Expression
}

\author{
Hongping Xia ${ }^{1,2,3} \mathbb{D} \cdot$ Yinhua Liu ${ }^{4} \cdot$ Zhichun Wang $^{1} \cdot$ Wei Zhang ${ }^{4} \cdot$ Min $\mathrm{Qi}^{1} \cdot$ Bin $\mathrm{Qi}^{5} \cdot$ Xiaochun Jiang $^{1}$
}

Published online: 5 November 2019

(C) The American Society for Experimental NeuroTherapeutics, Inc. 2019

\begin{abstract}
Noncoding RNAs regulate transcription of gene expression and play an important role in the pathogenesis of glioblastomas. These tumors are heterogeneous with some glioma stem cells (GSCs) that are highly tumorigenic subpopulations of cells contributing to recurrence and treatment resistance. In this study, GSCs were established by neurosphere cultures of primary glioblastoma cells and validated by the expression of GSC marker CD133. The expression of the long noncoding RNA HOTAIRM1 was detected using real-time quantitative reverse transcription PCR (qRT-PCR). The role of HOTAIRM1 in the proliferation, apoptosis, stemness, and tumorigenicity of GSCs was investigated by soft agar colony formation, flow cytometry, TUNEL analysis, sphere formation, and in vivo xenograft models through silencing of HOTAIRM1. The expression of HOTAIRM1 and the neighboring HOX genes were analyzed by qRT-PCR in different grades of gliomas and nontumor tissues. We found that HOTAIRM1 is significantly elevated in GSCs. The silencing of HOTAIRM1 significantly impairs the proliferation, apoptosis, self-renewal, tumorigenesis of GSCs. In addition, HOTAIRM1 is significantly upregulated in gliomas and associated with tumor grade and patient survival. HOTAIRM1 neighboring genes, HOXA1, HOXA2, and HOXA3, are also significantly upregulated in gliomas and correlate with the expression of HOTAIRM1. Among them, HOXA2 and HOXA3 were identified as being upregulated in GSCs and contributed to the self-renewal of these stem cells. Taken together, our results demonstrate that HOTAIRM1 plays a critical role in the self-renewal of GSCs. These data also suggest that overexpression of HOTAIRM1 can be a negative prognostic factor for patient survival in malignant glioma and may be a promising potential therapeutic target.
\end{abstract}

Key Words Glioblastoma stem cells $\cdot$ CD133 $\cdot$ LncRNAs $\cdot$ HOTAIRM1 $\cdot$ HOX genes

Electronic supplementary material The online version of this article (https://doi.org/10.1007/s13311-019-00799-0) contains supplementary material, which is available to authorized users.

Hongping Xia

xiahongping@njmu.edu.cn

$\triangle$ Xiaochun Jiang

Jiangxiaochun2001@hotmail.com

1 Department of Neurosurgery, Yijishan Hospital, Wannan Medical College, Wuhu 241001, China

2 Department of Pathology, School of Basic Medical Sciences \& The Affiliated Sir Run Run Hospital \& State Key Laboratory of Reproductive Medicine \& Key Laboratory of Antibody Technique of National Health Commission, Nanjing Medical University, Nanjing 211166, China
3 Department of Surgery, First Affiliated Hospital of Wenzhou Medical University, Wenzhou 325000, China

4 Department of Pathology, Yijishan Hospital, Wannan Medical College, Wuhu 241001, China

5 Department of Neurosurgery, First Hospital of Jilin University, Changchun, Jilin 130021, China 


\section{Introduction}

Glioma is the most common malignant adult primary brain tumor type in the central nervous system (CNS). Glioma can be categorized into 4 grades according to the World Health Organization (WHO) grading system, including low-grade glioma (WHO I and II) and high-grade glioma (WHO III and IV). Glioblastoma is classified as WHO grade IV and is the most aggressive and deadly form of glioma. There were 23,880 brain and other nervous system cancer cases in the USA in 2018 [1] and 101,600 new brain and other nervous system cancer cases in China in 2015 [2]. Glioblastoma accounts for $\sim 50 \%$ of gliomas. The current standard treatment of glioblastoma is surgery, followed by concurrent chemotherapy with temozolomide (TMZ) and radiation therapy [3]. The median overall survival of glioblastoma is $\sim 15$ months, and the 5-year survival rate is $\sim 5.5 \%$ [4]. Therefore, it is critical to investigate further the potential therapeutic targets for gliomas.

Glioma stem cells (GSCs) within high-grade gliomas are considered to contribute to treatment resistance and recurrence of glioblastoma. Their ability to grow as neurospheres in serum-free media has been used to enrich glioma GSCs. CD133 is the most commonly used cell surface marker of GSCs. CD133 (prominin-1)+ is considered to have higher rates of self-renewal and proliferation and increased differentiation ability. Injection of a few CD133+ glioma cells can produce a tumor in the brain of a mouse, which could be serially transplanted with a phenotype copy of the patient's original tumor [5]. GSCs have been considered to play a crucial role in resistance to conventional therapies and the initiation of tumor recurrence [6]. Therefore, the identification and understanding of GSCs will provide insights into human glioma pathogenesis for developing more effective therapeutic strategies.

Long noncoding RNAs (LncRNAs) are a class of RNA that are longer than $200 \mathrm{nt}$ and lack protein-coding capacity. lncRNAs are emerging as important regulators of gene expression, and contribute to normal cell and tissue physiology and disease processes including cancer pathogenesis [7]. Recent research indicates that the aberrant expression of LncRNAs also plays an important role in the self-renewal and malignant transformation of cancer stem cells, including GSCs. LncRNAs may become novel biomarkers for diagnosis and therapeutic targets for the treatment of glioma $[8,9]$. However, there is no functional study of HOTAIRM1 in glioma, especially glioma stem cells. Here, we discovered that HOTAIRM1 is elevated in GSCs and plays a critical role in the self-renewal of GSCs. The upregulation of HOTAIRM1 is a negative prognostic factor for patient survival in malignant glioma and may be a potentially promising therapeutic target.

\section{Materials and Methods}

See the Supplementary Material for a complete description of the methods.

\section{Sphere Formation Assay}

Established GSC1 and GSC2 cells were infected with lentiviral shHOTAIRM1 or shRNA scramble and pLentiHOTAIRM1, pLenti-HOXA2/HOXA3, or pLenti-control virus. Cells were plated onto a 24-well ultralow attachment plate (Corning Inc., Corning, NY.) in neurosphere culture medium, which consisted of serum-free sphere condition media containing DMEM/F $12,10 \mathrm{ng} / \mathrm{mL}$ basic fibroblast growth factor (bFGF), $10 \mathrm{ng} / \mathrm{mL}$ epidermal growth factor (EGF), 1:50 B27 supplement, and $100 \mathrm{U} / \mathrm{mL}$ penicillin/streptomycin (Thermo Fisher Scientific, Rockford, IL.). After 2 to 3 weeks of culture, the number of neurospheres (diameter $>40 \mu \mathrm{m}$ ) was manually counted in 3 randomly selected fields at a magnification of $40 \times$ under an inverted microscope. This assay was performed in triplicate in 3 independent experiments.

\section{Orthotopic Xenograft Models}

Animal experiments were conducted according to the regulations of the Laboratory Animal Research Center at the institute. To establish the orthotopic xenografts of GBM, 5week-old NOD/SCID mice were used. GSC1 and GSC2 cells were infected with lentiviral Luciferase and lentiviral shHOTAIRM1 or shRNA scramble. Then $2 \times 10^{5}$ stable infected cells were collected in $10 \mu \mathrm{L}$ of Hank's balanced salt solution for a group of 5 mice and injected into the brains of anesthetized mice ( $2 \mu \mathrm{L} /$ mice $)$ by using a rodent stereotactic frame. We used $\mathrm{X}$ and $\mathrm{Y}$ stereotaxic coordinates and positioned the micropipette over the area to be injected. The tumor growth was monitored by bioluminescence imaging at different time points. The animals were sacrificed using asphyxiation by $\mathrm{CO}_{2}$ or via exsanguination under deep isoflurane anesthesia and the tumor volume was examined.

\section{Statistics}

All data and statistics analysis was performed using ANOVA and a two-tailed Student's $t$ test as implemented in SPSS 12.0, GraphPad Prism 5 software or Partek Genomics Suite software. A $p$ value $<0.05$ was considered significant, and the results were adjusted for multiple comparisons. 


\section{Results}

\section{HOTAIRM1 is Elevated in GSCs, and the Suppression of HOTAIRM1 Impairs Self-Renewal}

We previously established some GSCs from fresh surgical samples of GBM patients and found that some microRNAs and lncRNAs are dysregulated in GSCs and associated with stemness of glioblastoma. We found that the established GSCs have a significantly high neurosphere formation capacity compared to non-GSCs, which were primary-cultured GBM cells in the normal culture medium with the adherent culture condition (Fig. 1a). We further examined the expression of CD133 by qRT-PCR and flow cytometry analysis. The results also showed that the normalized relative expression of the GSC marker CD133 (PROM1 gene) was significantly higher in GSCs than in non-GSCs (Fig. 1b). The CD133+ GSC subpopulations were also significantly higher in the neurospherecultured GSCs than in the established primary GBM cells by flow cytometry analysis (Fig. 1c). Some other GSC stemness genes (SOX2, OCT4, NANOG) are also higher in GSCs than non-GSCs (Fig. S11A-C). Next, we examined a panel of lncRNAs expression in the GSCs and non-GSCs. We found that HOTAIRM1 is elevated in GSCs (Fig. 1d). To further investigate the role of HOTAIRM1 in GSCs, we designed 3 shRNAs targeting HOTAIRM1. After the transfection of GSCs, we observed significant silencing of HOTAIRM1 expression in GSCs by qRT-PCR analysis (Fig. 1e). Interestingly, the silencing of HOTAIRM1 also significantly inhibits the neurosphere-forming ability of GSCs (Fig. 1f), suggesting that suppression of HOTAIRM1 impairs the selfrenewal of GSCs.

\section{Silencing of HOTAIRM1 Inhibits the Proliferation and Tumorigenicity of GSCs}

To investigate the critical role of HOTAIRM1 in GSCs, we further investigated the effects of HOTAIRM1 silencing on cell growth, proliferation, and tumorigenic capacity of GSCs. We found that the silencing of HOTAIRM1 significantly inhibits the cell growth of GSCs by MTS (3-(4,5-dimethylthiazol-2-yl)-5-(3-carboxymethoxyphenyl)-2-(4sulfophenyl)-2H-tetrazolium) analysis (Fig. 2a, b). The trypan blue exclusion viability assay also showed the inhibition effects of HOTAIRM1 shRNA (Fig. S12E). The MTS assay is a colorimetric method for the sensitive quantification of viable cells in cell proliferation assays. Anchorage-independent growth in soft agar is the ability of transformed cells to grow independently and is a hallmark of carcinogenesis. To characterize the proliferation and carcinogenesis capability of GSCs in vitro, we next conducted a soft agar colony formation assay.
The results showed that the silencing of HOTAIRM1 in GSCs formed far fewer colonies than in the control group, and the size of the colonies was also much smaller than that of the control group (Fig. 2c, d). Our data also demonstrated that reoverexpression of HOTAIRM1 could restore most of the in vitro functions mediated by HOTAIRM1 shRNA1 (Fig. S1). Moreover, HOTAIRM1 overexpression in the "naïve"/ nonmanipulated GSCs (non-GSC1 and non-GSC2) that express low HOTAIRM1 also showed that overexpression of HOTAIRM1 promotes non-GSC cell proliferation (Fig. S1E, F) and tumor growth (Fig. S1G, H). We also investigated the sensitivity of GSCs/non-GSCs and GSCs-ShScramble/ ShHOTAIRM1 to temozolomide (TMZ). The results showed that GSCs are more resistant to TMZ. However, knockdown of HOTAIRM1 significantly overcomes the TMZ resistance of GSCs (Fig. S12A-D). The tumorigenicity assay is considered the gold standard assay for cancer stem cells. So we next examined the tumorigenic capacity of GSCs through silencing HOTAIRM1. We found that the silencing of HOTAIRM1 significantly inhibited the tumorigenic capacity of GSCs in vivo (Fig. $2 \mathrm{E}-\mathrm{H}$ ), suggesting that HOTAIRM1 is critical for the proliferation and tumorigenicity of GSCs.

\section{Silencing of HOTAIRM1 Induces Apoptosis of GSC Cells}

To understand how HOTAIRM1 contributes to the proliferation and tumorigenicity of GSCs, we investigated the effects of HOTAIRM1 silencing on the apoptosis of GSCs. An Annexin/7-AAD staining assay showed that suppression of HOTAIRM1 in both GSC1 and GSC2 cells significantly increased the Annexin+ apoptotic cells (2-3 fold) by flow cytometry analysis (Fig. 3a). Meanwhile, the TUNEL assay results also showed that suppression of HOTAIRM1 significantly increased the TUNEL+ apoptotic cell number (Fig. 3b). These data suggest that the silencing of HOTAIRM1 may inhibit the proliferation and tumorigenicity of GSCs through inducing apoptosis of GSCs.

\section{HOTAIRM1 is Overexpressed in Gliomas and is a Negative Prognostic Factor for Patients}

To evaluate the critical role of HOTAIRM1 as a potential biomarker of GBM, we next examined the expression of HOTAIRM1 in glioma tissues from glioma patients of different grades by qRT-PCR analysis. We observed that the expression of HOTAIRM1 was significantly higher in gliomas than in nontumor brain tissues (Fig. 4a). Moreover, the expression level of HOTAIRM1 correlated with the grades of gliomas. The expression of HOTAIRM1 was significantly higher in grade 4 glioma (GBM) than in grade 2 and grade 3 gliomas (Fig. 4b). There was also a 
a

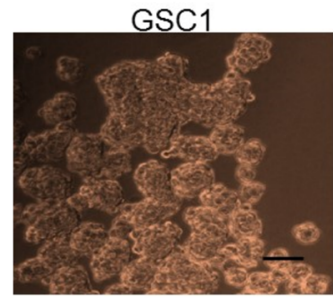

GSC2

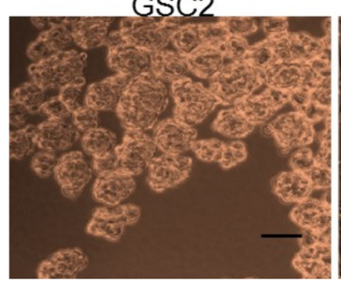

C

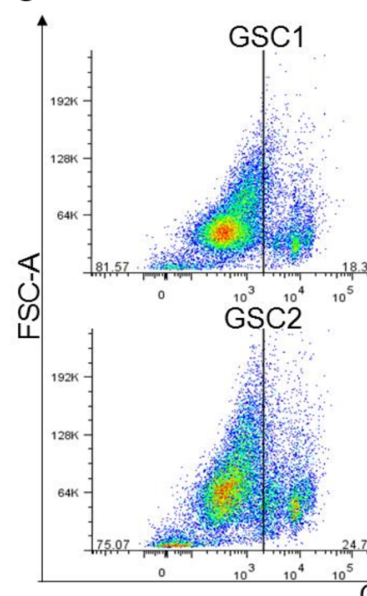

Non-GSC1

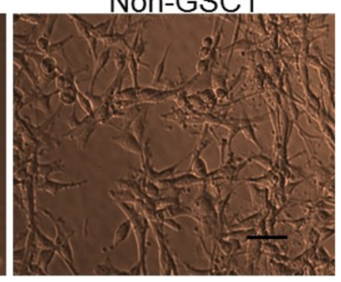

Non-GSC2
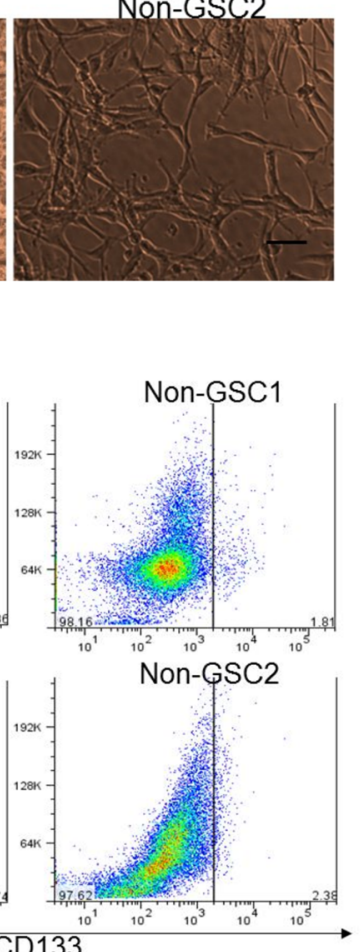

b

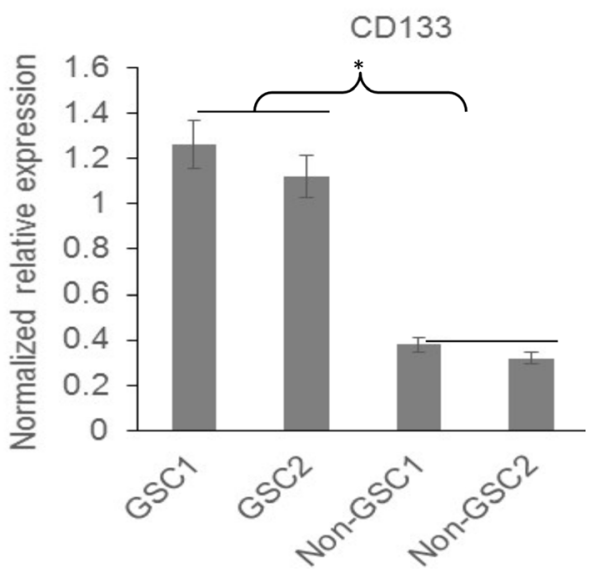

d

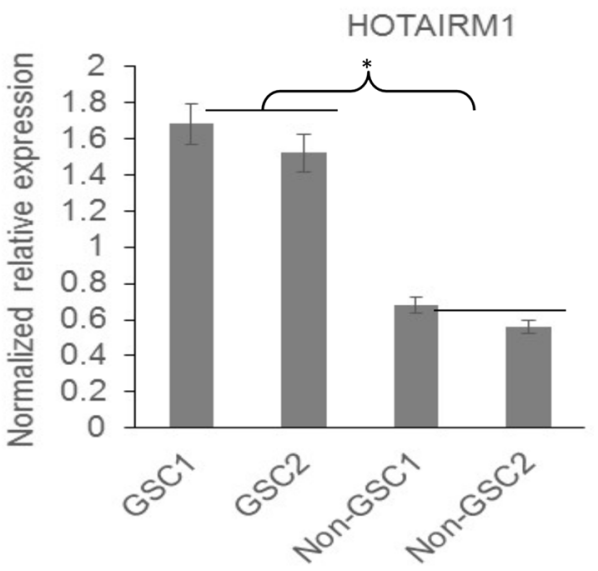

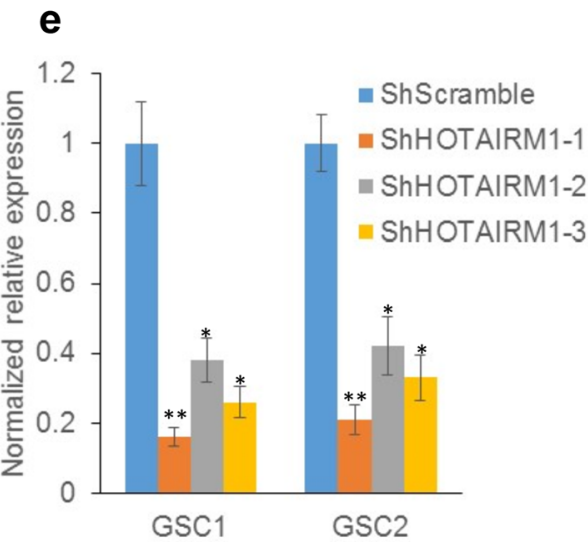

Fig. 1 HOTAIRM1 is elevated in GSCs and suppression of HOTAIRM1 impairs self-renewal. (a) The representative images of neurospherecultured GSCs and 2 established GSC cells. (b) The normalized relative expression of the GSC marker CD133 was significantly higher in GSCs than in non-GSCs by qRT-PCR analysis. (c) The CD133+ GSC subpopulations were significantly higher in the neurosphere-cultured GSCs than in the established primary glioblastoma cells by flow cytometry analysis. f

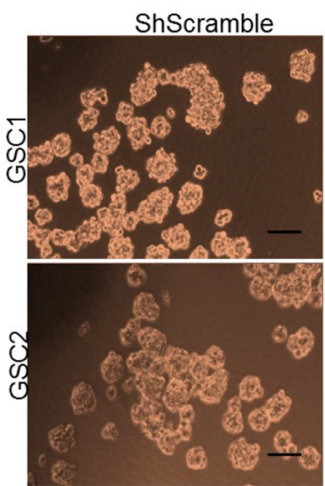

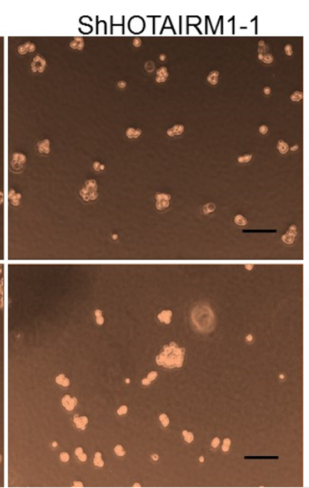

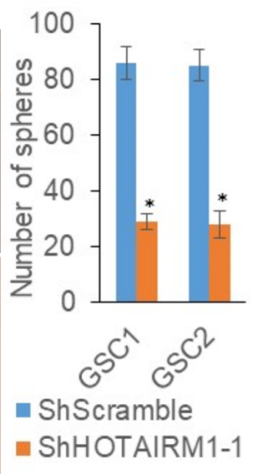

(d) The normalized relative expression of HORTAIRM1 was significantly higher in the GSCs than in the non-GSCs by qRT-PCR analysis. (e) The knockdown effects of 3 different HOTAIRM1 shRNA-infected GSCs were validated by qRT-PCR analysis. (f) The representative images of the sphere formation in GSCs infected with ShScramble and HOTAIRM1 shRNA. An asterisk (*) denotes $p<0.05$, whereas a double asterisk $(* *)$ denotes $p<0.01$ )

and noncodeletion (Fig. 4c, d). The median relative expression value of grade 4 or grade $2 / 3$ glioma tissue samples

significant difference in HOTAIRM1 expression between the IDH1 mutation and wild-type or the $1 \mathrm{p} / 19 \mathrm{q}$ codeletion 
Fig. 2 Silencing of HOTAIRM1 inhibits the proliferation and tumorigenicity of GSCs. $(\mathbf{a}, \mathbf{b})$ The cell growth rate was significantly inhibited in HOTAIRM1 shRNA-infected GSCs using MTS assay analysis. (c, d) The representative images and quantification of colonies showed that the silencing of HOTAIRM1 inhibits the colonyforming ability of GSCs in soft agar. (e, f) Tumor growth in the xenograft models was significantly promoted in the HOTAIRM1-silenced GSC group compared to the ShScrambleinfected GSC control group. An asterisk (*) denotes $p<0.05$, whereas a double asterisk $(* *)$ denotes $p<0.01)$. (g, h) The representative bioluminescence imaging signal of tumor growth in the xenograft models of the ShScramble- and ShHOTAIRM1-infected GSC control group a

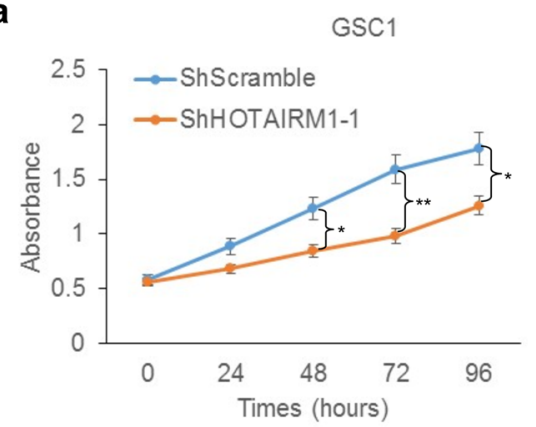

C
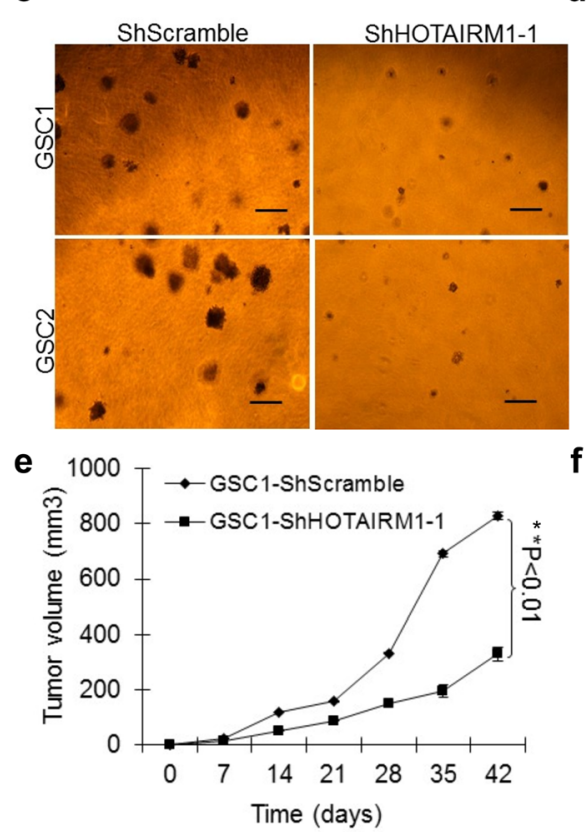

g

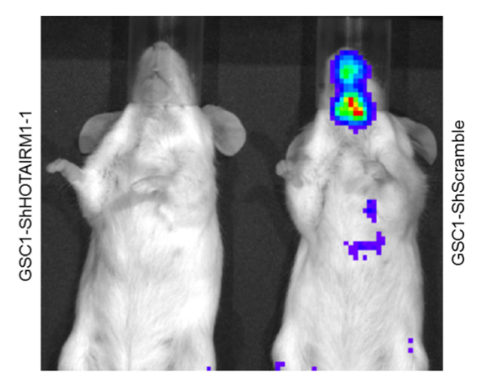

b

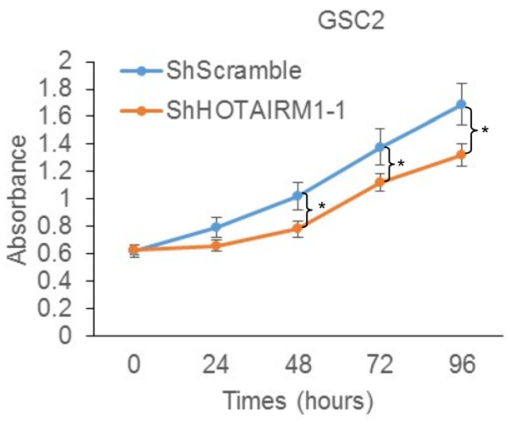

d
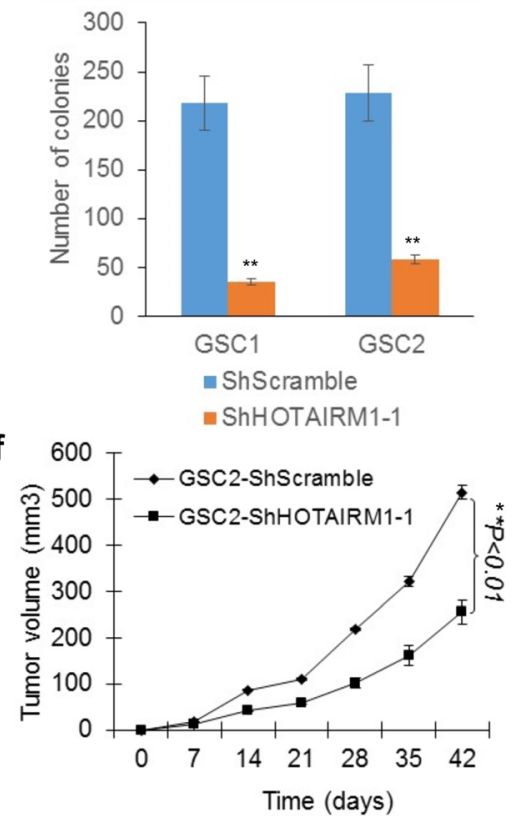

h

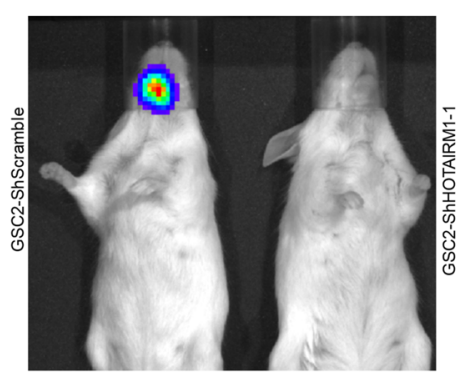

was chosen as the cutoff point. Fisher's exact test and Kaplan-Meier analysis showed that a high level of HOTAIRM1 expression was significantly associated with poor overall survival of both grade 4 glioma (Fig. 4e) and grade $2 / 3$ glioma (Fig. 4f). The expression of HOTAIRM1 was further shown to be elevated with advanced stages of gliomas in the Chinese Glioma Genome Atlas (CGGA) data set (Fig. 4g). The high expression of HOTAIRM1 was also significantly associated with the overall survival of gliomas in the CGGA data set (Fig. 4h). The GSE4290 data set also showed that HOTAIRM1 is overexpressed in gliomas compared to nontumor tissues (Fig. S2A). The
TCGA data set showed that the expression level of HOTAIRM1 was higher in GBM than in low-grade gliomas (LGGs) (Fig. S2B). However, the expression of HOTAIRM1 in 4 molecular subtypes of glioma (classical, mesenchymal, neural, and proneural) was not significantly different in the CGGA and TCGA data sets (Fig. S3). High expression of HOTAIRM1 was also significantly associated with poor survival of both GBM and LGG patients in the TCGA and CGGA data sets (Figs. S4 and S5). Multivariable analyses of the clinico-pathological parameters (age, gender, preoperative KPS, treatment, IDH1 mutation, MGMT methylation, and HOTAIRM1) of overall 
Fig. 3 Silencing of HOTAIRM1induced apoptosis of GSC cells.

(a) The Annexin/7-AAD staining assay showed that the knockdown of HOTAIRM1 significantly increased the Annexin+ apoptotic cells by flow cytometry analysis. (b) The representative images of the TUNEL assay showed that the knockdown of HOTAIRM1 significantly increased the TUNEL+ apoptotic cell number a

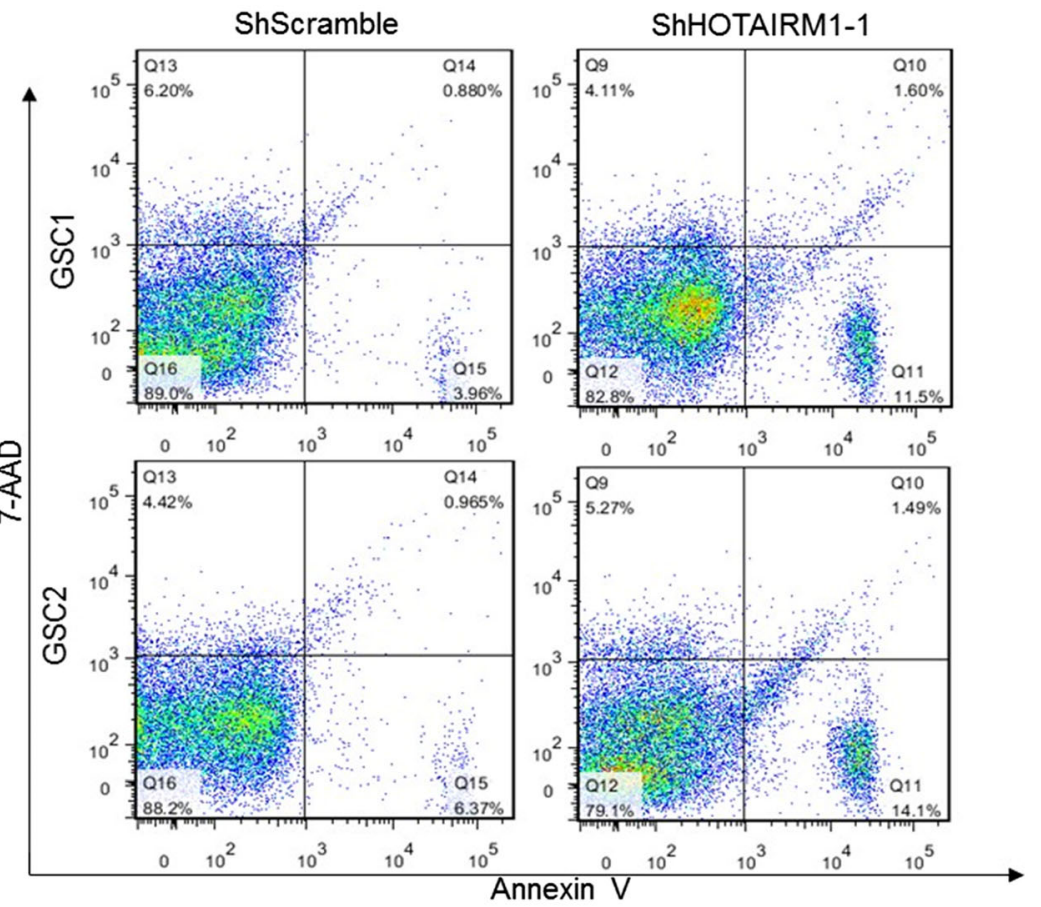

b

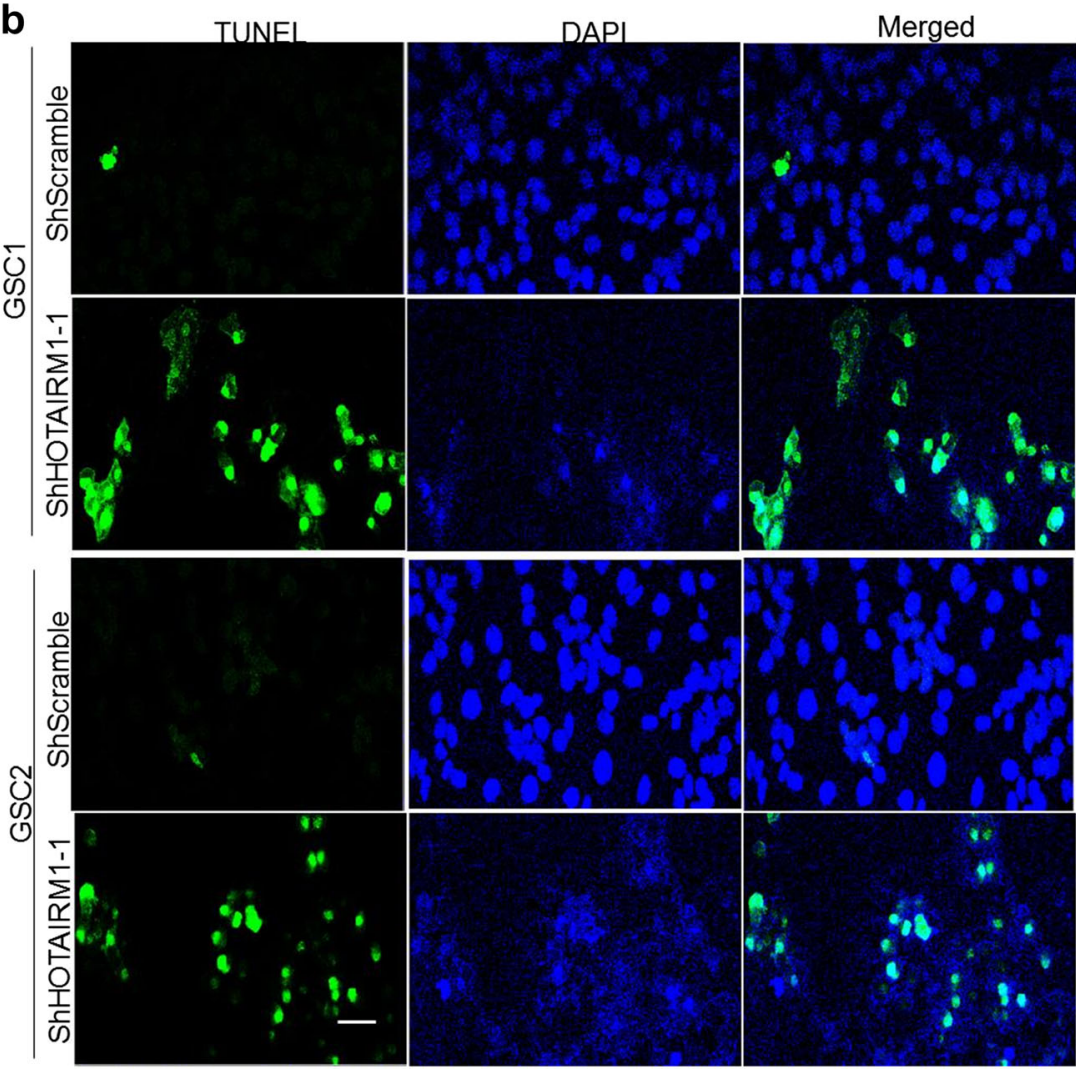

survival in patients with GBM and LGG have been done to establish HOTAIRM1 as an independent prognostic biomarker (Table S1). Overall, these data suggest that HOTAIRM1 is elevated in gliomas and is a negative prognostic factor for patients.

\section{The Neighboring Genes of HOTAIRM1 are Upregulated in GBM and Correlate with HOTAIRM1}

Emerging evidence suggested that many lncRNAs act locally to regulate the expression of nearby genes [10]. We observed 3 
Fig. 4 HOTAIRM1 is overexpressed in GBM and is a negative prognostic factor for patients. (a) The expression of HOTAIRM1 was significantly increased in gliomas compared to adjacent nontumor brain tissues by qRT-PCR analysis. (b) The expression of HOTAIRM1 was increased with the increase in the grades of the gliomas. The legends of axes are normalized expression relative to the housekeeping gene GAPDH by RTqPCR. (c, d) There was a significant difference in HOTAIRM1 expression between the IDH1 mutation and wild-type (c) or the $1 \mathrm{p} / 19 \mathrm{q}$ codeletion and noncodeletion (d). (e, f) The high expression of HOTAIRM1 was significantly associated with the overall survival of both grade IV glioblastoma (e) and grade 2/3 gliomas (f). (g) The expression of HOTAIRM1 was shown to be increased with the increase in the glioma grade in the Chinese Glioma Genome Atlas (CGGA) data set (total 325 samples: 109 grade 2, 72 grade 3, and 144 grade 4$)$. The legend of axes is the CGGA_Gene_Expression Profile_FPKM value by RNAseq. (H) The high expression of HOTAIRM1 was validated to be significantly associated with the overall survival of gliomas in the CGGA data set $\mathbf{a}_{\text {. }}$

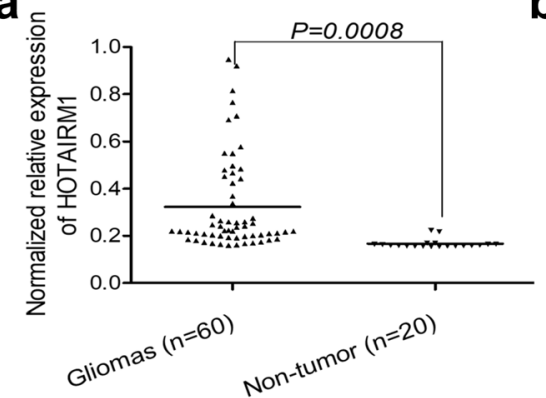

C
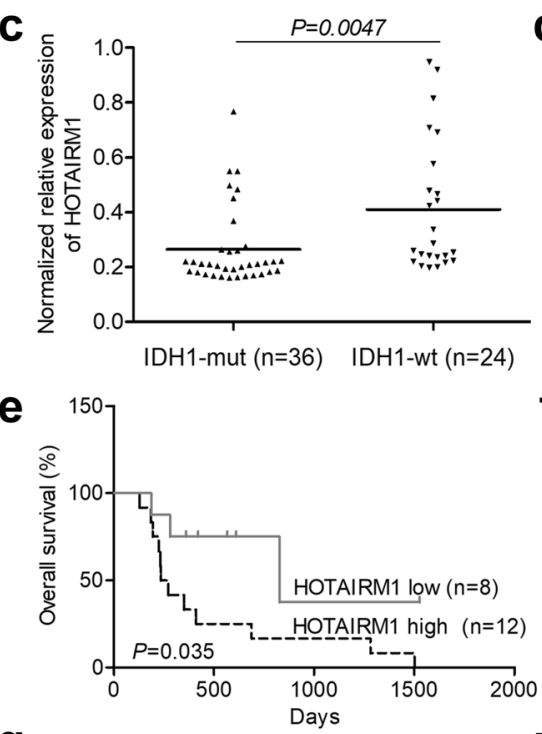

g

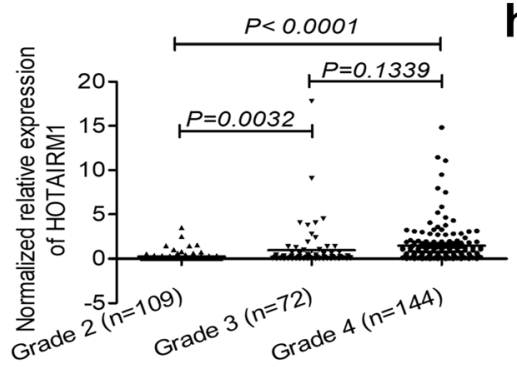

b

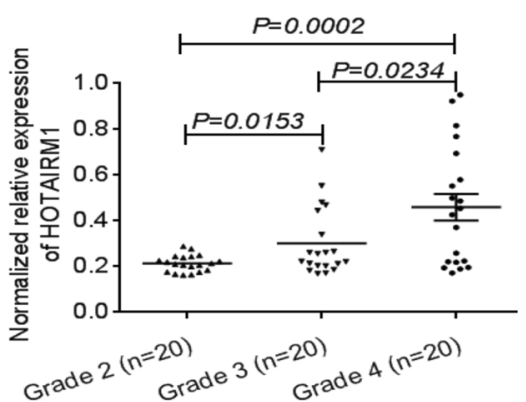

d

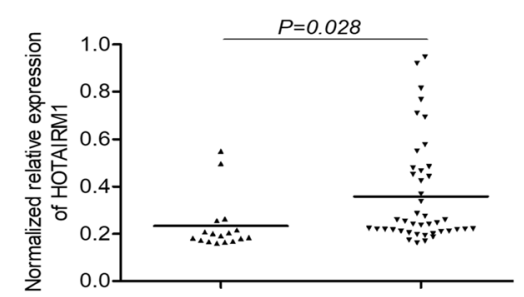

$1 p / 19 q$ Codel $(n=17)$ Np/19q Non-Codel $(n=43)$

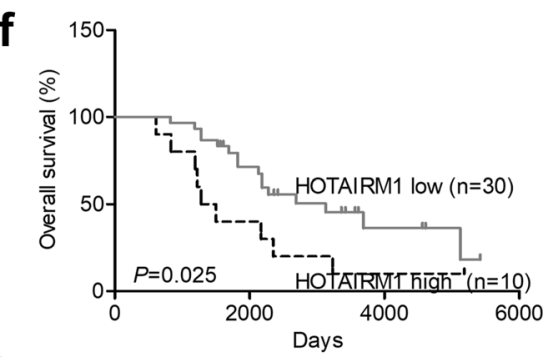

$h$

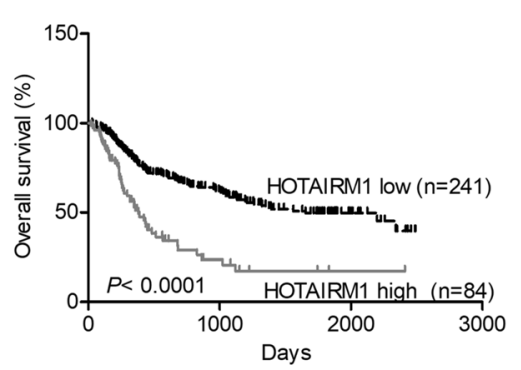

neighboring genes (HOXA1, HOXA2, and HOXA3) of HOTAIRM1 by bioinformatics analysis (Fig. S6). Next, we examined the expression of neighboring genes in our samples by qRT-PCR analysis. We found that the expression of HOXA1, HOXA2, and HOXA3 was significantly increased in gliomas and correlated with the expression of HOTAIRM1 (Fig. 5). Pan-cancer analysis of HOXA1, HOXA2, and HOXA3 in the TCGA data set also showed that HOXA1, HOXA2, and HOXA3 had a low expression in the normal brain but significantly overexpressed in both GBM and LGGs (Fig. S7). The correlations of HOTAIRM1 and HOXA genes in the TCGA are shown in Fig. S8. There was also a significant difference in HOXA1, HOXA2, and HOXA3 expression between the IDH1 mutation and wildtype or the 1p/19q codeletion and noncodeletion (Fig. S9). Although HOXA9 and HOXA10 have been established as critical oncogenes in GBM, we did not observe a difference in the expression of HOXA9 and HOXA10 in our GSCs and non-GSCs by RT-qPCR analysis (Fig. S11D, E). We next performed a knockdown assay of HOXA1, HOXA2, or HOXA3. The results showed that the knockdown of HOXA1, HOXA2, or HOXA3 inhibited colony formation in soft agar of GSCs independent of HOTAIRM knockdown, which suggests the effects of HOXA gene-silencing on GSC tumorigenicity (Fig. S10).

\section{HOXA2 and HOXA3 are Upregulated in GSCs and Contribute to the Self-Renewal of GSCs}

To further investigate the role of neighboring genes of HOTAIRM1 in the self-renewal of GSCs, we firstly examined the expression of HOXA1, HOXA2, and 
Fig. 5 The neighboring genes of HOTAIRM1 were upregulated in GBM and correlated with HOTAIRM1. (a, b) The expression of HOXA1 was significantly increased in gliomas $(p<0.0006)$ and correlated with the expression of HOTAIRM1 (Pearson $r=0.77, p<0.0001)$. (c, d) The expression of HOXA2 was significantly increased in gliomas $(p=0.0099)$ and correlated with the expression of HOTAIRM1 (Pearson $r=0.73, p$ $<0.0001)$. (e, f) The expression of HOXA3 was significantly increased in gliomas $(p<0.0001)$ and correlated with the expression of HOTAIRM1 (Pearson $r=0.84$, $p<0.0001)$ a

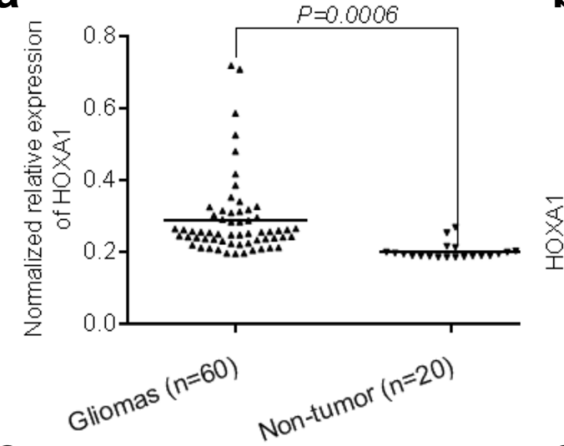

C

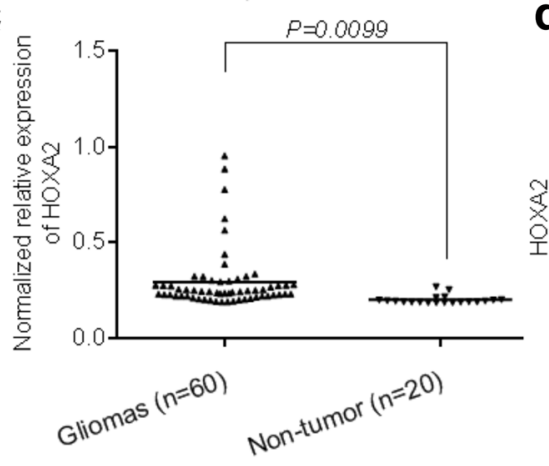

e

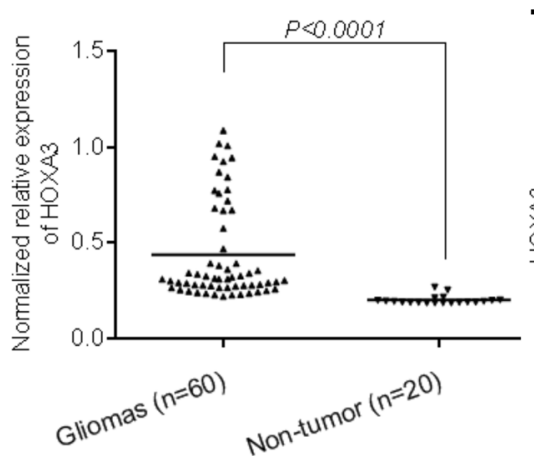

b

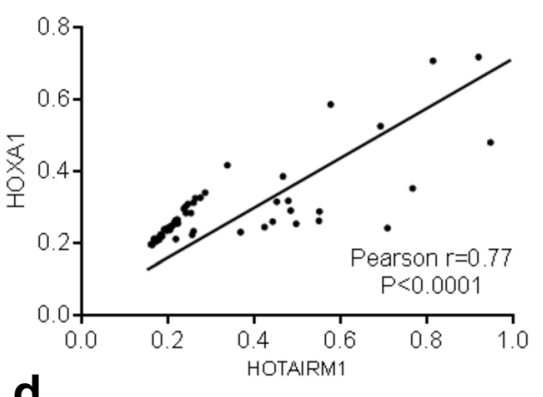

d

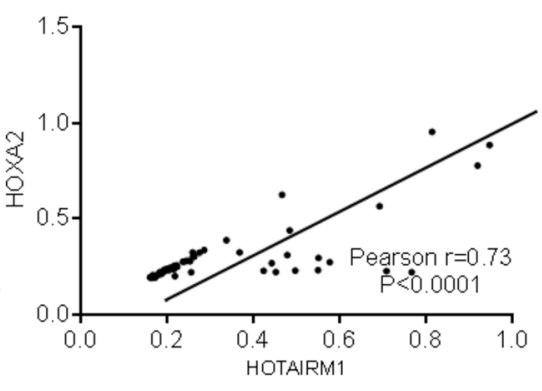

f

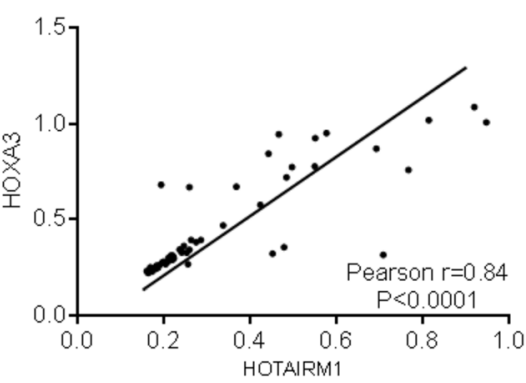

HOXA3 in GSCs and non-GSCs and GSCs with the knockdown of HOTAIRM1 by qRT-PCR analysis. The normalized relative expression of HOXA1, HOXA2, and HOXA3 was downregulated in GSCs with the suppression of HOTAIRM1 (Fig. 6a). However, the expression of HOXA2 and HOXA3, but not HOXA1, was significantly higher in GSCs than in non-GSCs (Fig. 6b). To further understand the role of HOXA2 and HOXA3 in the self-renewal of GSCs, we next force-expressed the expression of HOXA2 and HOXA3 in GSCs with the silencing of HOTAIRM1. The quantification of the neurospheres showed that overexpression of HOXA2 and HOXA3 significantly increased the neurosphereforming ability of GSCs when HOTAIRM1 was silenced (Fig. 6c, d), suggesting that HOXA2 and HOXA3 were upregulated in GSCs and contribute to the self-renewal of GSCs induced by HOTAIRM1.

\section{Discussion}

HOTAIRM1, HOXA transcript antisense RNA myeloidspecific 1 , is a novel long noncoding RNA that has been found to play a role in myelopoiesis through modulation of gene expression of the HOXA cluster [11]. The following study showed that HOTAIRM1 provides a regulatory link during myeloid maturation in NB4 human promyelocytic leukemia cells [12] by modulating integrin-controlled cell cycle progression at the gene expression level. Recently, HOTAIRM1 has been found to play a role in some cancers, including breast cancer [13], acute myeloid leukemia [14], pancreatic ductal adenocarcinoma [15], ovarian cancer [16], lung adenocarcinoma [16], head and neck tumors [17], and colorectal cancer (CRC) [18]. In breast cancer, HOTAIRM1 was found to be significantly overexpressed in the basal-like subgroup whereas HOTAIR was significantly overexpressed in the HER2- 
Fig. 6 HOXA2 and HOXA3 were upregulated in GSCs and contributed to the self-renewal of GSCs. (a) The normalized relative expression of HOXA1, HOXA2, and HOXA3 in GSC1 cells with knockdown of HOTAIRM1 by qRT-PCR analysis. (b) The normalized relative expression of HOXA1, HOXA2, and HOXA 3 in GSCs and nonGSCs by qRT-PCR analysis. (c) The normalized relative expression of HOXA2 and HOXA3 showed overexpression of HOXA 2 and A3 by qRT-PCR analysis. (d, e) The representative images and quantification of neurospheres showed that overexpression of HOXA2 and HOXA3 significantly increased the neurosphere formation ability of GSCs with the silencing of HOTAIRM1. An asterisk (*) denotes $p<0.05$ a

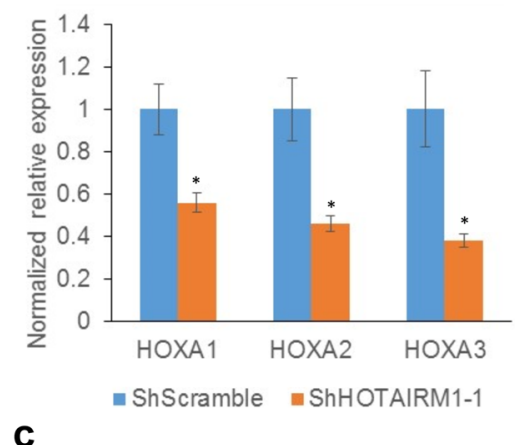

C

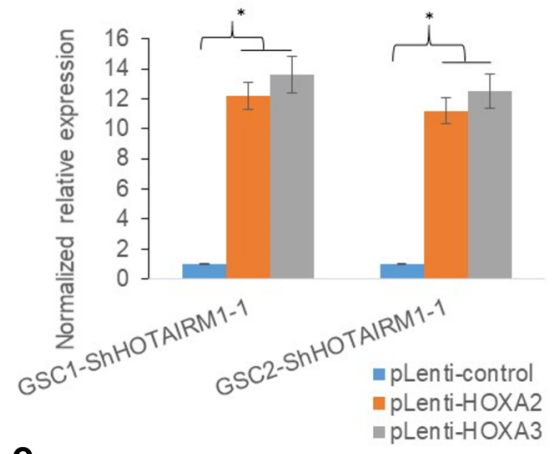

b

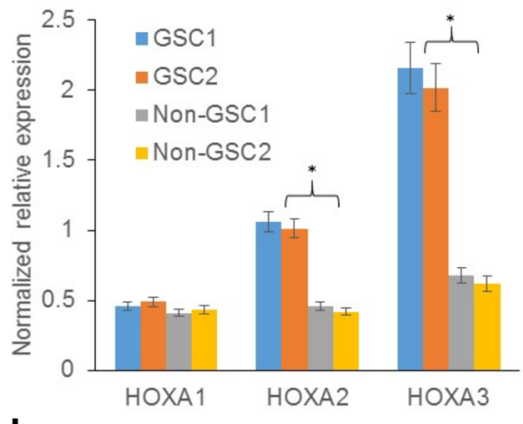

d

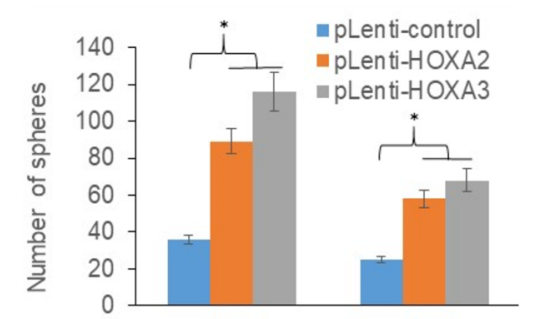

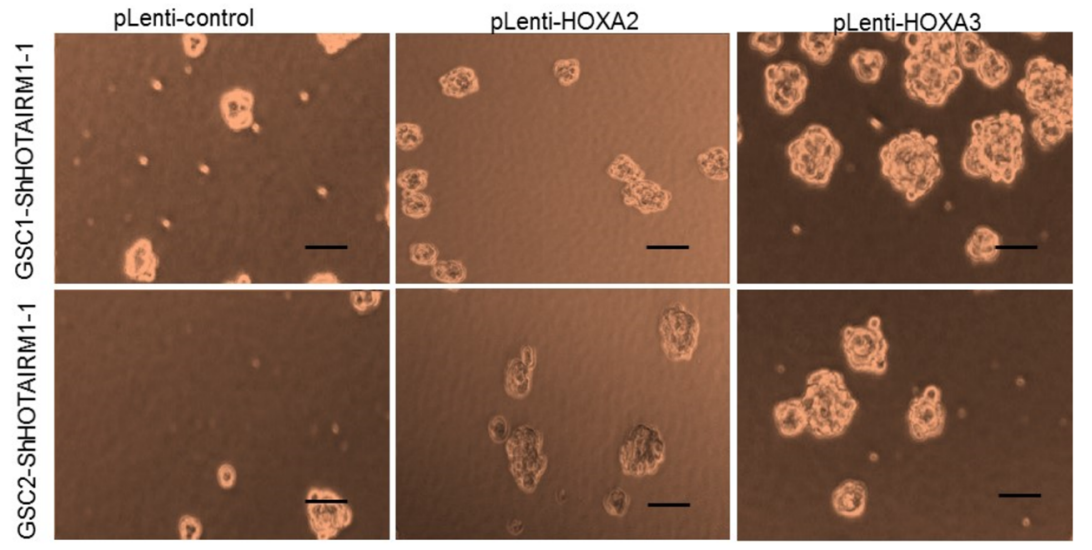

enriched subgroup [13]. In intermediate-risk acute myeloid leukemia patients, high HOTAIRM1 expression was independently associated with shorter overall survival, shorter leukemia-free survival, and a higher cumulative incidence of relapse [14]. HOTAIRM1 has also been shown to regulate autophagy and the degradation of the PML-RARA oncoprotein during myeloid cell differentiation [19]. The expression level of HOTAIRM1 was upregulated in pancreatic ductal adenocarcinoma tissue samples compared with matched adjacent nontumor samples [15]. In different types of lung cancer, HOTAIRM1 was found to be mainly expressed in lung adenocarcinoma. However, HOTAIRM1 was decreased in the peripheral blood cells of lung cancer patients compared with those of healthy controls [20]. The expression of HOTAIRM1 was also found to be reduced in CRC tissues compared with matched normal tissues, and plasma HOTAIRM1 levels in CRC patients were also lower than those of controls, suggesting that HOTAIRM1 plays the role of a tumor suppressor in CRC [18].

Previously, differential lncRNA expression profiles in recurrent gliomas compared with primary gliomas also indicated that some lncRNAs, such as H19, CRNDE, and HOTAIRM1, may play important roles in glioma recurrence $[9,21]$. GSCs are considered a source of tumor formation and recurrence of glioma. However, the critical role of HOTAIRM1 in the pathogenesis and stemness of glioma has not been clarified. Here, we discovered that HOTAIRM1 is significantly elevated in GSCs. The silencing of HOTAIRM1 significantly impairs the proliferation, apoptosis, self-renewal, and tumorigenesis of GSCs, suggesting the critical role of HOTAIRM1 in the stemness of GSCs. HOTAIRM1 was significantly overexpressed in glioma tissue samples and was 
associated with the grading of the tumors and patients' survival. The TCGA data indicated that the high expression level of HOTAIRM1 was a negative prognostic factor for both GBM and LGGs. It is expected to be a negative prognostic factor for all glioma patients irrespective of tumor grade. The overexpression of HOTAIRM1 was a negative prognostic factor for patient survival in malignant glioma and may be a potentially promising therapeutic target.

Emerging evidence suggested that many lncRNAs act locally to regulate the expression of nearby genes [10]. For a further understanding of the regulatory mechanism of HOTAIRM1 in the pathogenesis of glioma, we further investigated the expression and function of 3 neighboring genes (HOXA1, HOXA2, and HOXA3) of HOTAIRM1. The expression of HOXA1, HOXA2, and HOXA3 was significantly increased in gliomas and correlated with the expression of HOTAIRM1. A previous study showed that chromosome 7 and DNA hypermethylation is associated with the expression of a stem cell-related HOX signature in GBM. HOX genes are a family of developmental genes that are not expressed in the developing forebrain and normal adult brain. The aberrant expression of a HOX genedominated stem cell signature in GBM has been linked with increased resistance to chemoradiotherapy and sustained proliferation of GSCs [22]. HOXA1 has been identified as a putative driver of early mammary cancer progression in transgenic mice. Silencing of HOXA1 by intraductal injection of siRNA lipidomic nanoparticles has been shown to prevent mammary tumor progression in mice [23]. HOXA2 has been shown to select barrelette neuron identity and connectivity in the mouse somatosensory brainstem [24]. HOXA3 has been shown to promote tumor growth of colon cancer through activation of the EGFR/Ras/Raf/MEK/ERK signaling pathway [25]. Our data indicated that HOXA2 and HOXA3 were further shown to be upregulated in GSCs and contribute to the self-renewal of GSCs induced by HOTAIRM1. Therefore, our data demonstrated that HOTAIRM1 maintains the tumorigenicity of GSCs through regulation of the HOX gene expression.

Acknowledgments This study was supported by grants from the National Natural Science Foundation of China, the Anhui Province Education Department, the National Young 1000 Talents Program of China, the Jiangsu Province Education Department grant, and the Jiangsu Province "Innovative and the Entrepreneurial Team" and "Innovative and Entrepreneurial Talent."

Required Author Forms Disclosure forms provided by the authors are available with the online version of this article.

\section{Compliance with Ethical Standards}

Conflict of Interest The authors declare that they have no competing interests.

\section{References}

1. L. SR, D. MK, Ahmedin J. Cancer statistics, 2018. CA: a cancer journal for clinicians. 2018;68:7-30. https://doi.org/10.3322/caac. 21442.

2. Chen W, Zheng R, Baade PD, et al. Cancer statistics in China, 2015. CA: a cancer journal for clinicians. 2016;66(2):115-132.

3. Lim M, Xia Y, Bettegowda C, Weller M. Current state of immunotherapy for glioblastoma. Nature Reviews Clinical Oncology. 2018;15(7): 422-442.

4. Nizamutdinov D, Stock EM, Dandashi JA, et al. Prognostication of survival outcomes in patients diagnosed with glioblastoma. World Neurosurgery. 2018;109:e67-e74.

5. Singh SK, Hawkins C, Clarke ID, et al. Identification of human brain tumour initiating cells. Nature. 2004;432:396.

6. Osuka S, Van Meir EG. Overcoming therapeutic resistance in glioblastoma: the way forward. The Journal of Clinical Investigation. 2017;127:415-426.

7. Iyer MK, Niknafs YS, Malik R, et al. The landscape of long noncoding RNAs in the human transcriptome. NatureGenetics. 2015;47:199.

8. Peng Z, Liu C, Wu M. New insights into long noncoding RNAs and their roles in glioma. Molecular Cancer. 2018;17:61.

9. Zhang X, Sun S, Pu JKS, et al. Long non-coding RNA expression profiles predict clinical phenotypes in glioma. Neurobiology of Disease. 2012;48:1-8. https://doi.org/10.1016/j.nbd.2012.06.004.

10. Joung J, Engreitz JM, Konermann S, et al. Genome-scale activation screen identifies a lncRNA locus regulating a gene neighbourhood. Nature. 2017;548:343.

11. Zhang X, Lian Z, Padden C, et al. A myelopoiesis-associated regulatory intergenic noncoding RNA transcript within the human HOXA cluster. Blood. 2009;113:2526-2534.

12. Zhang X, Weissman SM, Newburger PE. Long intergenic noncoding RNA HOTAIRM1 regulates cell cycle progression during myeloid maturation in NB4 human promyelocytic leukemia cells. RNA Biology. 2014;11:777-787.

13. Su X, Malouf GG, Chen Y, et al. Comprehensive analysis of long non-coding RNAs in human breast cancer clinical subtypes. Oncotarget. 2014;5:9864.

14. Díaz-Beyá $\mathrm{M}$, Brunet $\mathrm{S}$, Nomdedéu J, et al. The lincRNA HOTAIRM1, located in the HOXA genomic region, is expressed in acute myeloid leukemia, impacts prognosis in patients in the intermediate-risk cytogenetic category, and is associated with a distinctive microRNA signature. Oncotarget. 2015;6:31613.

15. Zhou Y, Gong B, Jiang Z-L, et al. Microarray expression profile analysis of long non-coding RNAs in pancreatic ductal adenocarcinoma. International Journal of Oncology. 2016;48:670-680.

16. Yang K, Hou Y, Li A, et al. Identification of a six-lncRNA signature associated with recurrence of ovarian cancer. Scientific Reports. 2017;7:752.

17. Zheng M, Liu X, Zhou Q, Liu G. HOTAIRM 1 competed endogenously with miR-148a to regulate DLGAP 1 in head and neck tumor cells. Cancer Medicine. 2018.

18. Wan L, Kong J, Tang J, et al. HOTAIRM 1 as a potential biomarker for diagnosis of colorectal cancer functions the role in the tumour suppressor. Journal of Cellular and Molecular Medicine. 2016;20: 2036-2044.

19. Chen Z-H, Wang W-T, Huang W, et al. The lncRNA HOTAIRM1 regulates the degradation of PML-RARA oncoprotein and myeloid cell differentiation by enhancing the autophagy pathway. Cell Death and Differentiation. 2017;24:212.

20. Tian X, Ma J, Wang T, et al. Long non-coding RNA HOXA transcript antisense RNA myeloid-specific 1-HOXA1 axis downregulates the immunosuppressive activity of myeloid- 
derived suppressor cells in lung cancer. Frontiers in Immunology. 2018;9:473.

21. Chen Y, Wu J-J, Lin X-B, et al. Differential lncRNA expression profiles in recurrent gliomas compared with primary gliomas identified by microarray analysis. International Journal of Clinical and Experimental Medicine. 2015;8:5033.

22. Kurscheid S, Bady P, Sciuscio D, et al. Chromosome 7 gain and DNA hypermethylation at the HOXA10 locus are associated with expression of a stem cell related HOX-signature in glioblastoma. Genome Biology. 2015;16:16.

23. Brock A, Krause S, Li H, et al. Silencing HoxA1 by intraductal injection of siRNA lipidoid nanoparticles prevents mammary tumor progression in mice. Science Translational Medicine. 2014;6: 217ra2-ra2.

24. Bechara A, Laumonnerie C, Vilain N, et al. HOXA2 selects barrelette neuron identity and connectivity in the mouse somatosensory brainstem. Cell Reports. 2015;13:783-797.

25. Zhang X, Liu G, Ding L, et al. HOXA3 promotes tumor growth of human colon cancer through activating EGFR/Ras/Raf/MEK/ERK signaling pathway. Journal of Cellular Biochemistry. 2018;119: 2864-2874.

Publisher's Note Springer Nature remains neutral with regard to jurisdictional claims in published maps and institutional affiliations. 